\title{
Pharmacy-Based Assessment and Management of Herpes Labialis (Cold Sores) with Antiviral Therapy
}

Alex J. Adams, PharmD, $\mathrm{MPH}^{1}$; Michael E. Klepser, PharmD, FCCP

${ }^{1}$ Idaho State Board of Pharmacy; ${ }^{2}$ Ferris State University, College of Pharmacy

\begin{abstract}
Herpes labialis, commonly known as cold sores, is an infection of the mouth and surrounding area. Antiviral therapy can be used to block viral replication, which shortens the duration of symptoms, facilitates resolution of lesions, and lessens the risk of spreading the virus. Increasing access to antivirals targeted against herpes labialis by allowing assessment and prescribing by a pharmacist may decrease time to treatment for HSV-1, and improve patient satisfaction. Experience from Canada, Australia, New Zealand and the United States demonstrate that pharmacist management of cold sores has a safe track record and may be considered by other jurisdictions.
\end{abstract}

Keywords: Pharmacy, Scope of Practice, Permissionless Innovation, Prescriptive

\section{Background}

Herpes labialis, commonly known as cold sores, is an infection of the mouth and surrounding area caused primarily by herpes simplex virus type 1 (HSV-1), and less commonly by herpes simplex virus type 2 (HSV-2). ${ }^{1}$ HSV-1 is a highly contagious virus that can be spread through direct or indirect contact with infected individuals. In the United States, there are approximately 500,000 primary infections with HSV-1 each year. ${ }^{2}$ It is estimated that by age 50 , about $90 \%$ of adults have been exposed to HSV $-1 .^{3} \quad$ Following the primary infection/episode with HSV-1 the virus is retained in the body and approximately $1 / 3$ of all infected individuals experience a relapse during their lifetime. ${ }^{4}$ Prior to experiencing a recurrent episode, prodromal symptoms (e.g., tingling, itching, burning) typically precede the formation of lesions. ${ }^{1}$ Infections are rarely serious and are typically self-limiting among immunocompetent hosts. Most lesions completely resolve within 2-4 weeks. $^{1}$

In the United States, the Food and Drug Administration (FDA) has approved several over-the-counter products as well as prescription-only antivirals for the episodic treatment of recurrent cold sores, both in topical and oral forms. ${ }^{1}$ Topical antiviral agents include penciclovir cream and acyclovir cream, while oral agents include acyclovir, famcyclovir, and valacyclovir. Antiviral therapy can be used to block viral replication, which shortens the duration of symptoms, facilitates resolution of lesions, and lessens the risk of spreading the virus. ${ }^{5}$ In order to realize maximum benefit, antiviral therapy is to be initiated as soon as possible following the onset of symptoms. Studies have demonstrated that use of antivirals can shorten the duration of and decrease pain associated with lesions. Some evidence suggests that oral medications maybe more effective than the topical agents. ${ }^{6}$

Corresponding author: Alex J. Adams, PharmD, MPH

Executive Director; Idaho State Board of Pharmacy

Boise, ID; Email: alexadamsrph@gmail.com
Oral antivirals can also be used prophylactically among those who experience frequent relapses. Topical agents are ineffective for prophylaxis since they do not penetrate to the site of reactivation. ${ }^{7}$

Although topical and systemic antivirals have been proven effective against HSV-1 and well tolerated, they are available only with a prescription in the United States. As a result, many patients do not have access to these medications in a timeframe that would optimize their benefits. Increasing access to antivirals targeted against herpes labialis by allowing assessment and prescribing by a pharmacist may decrease time to treatment for HSV-1, relieve congestion within the medical system, and improve patient satisfaction with their medical care. These benefits have been demonstrated for other acute, self-limiting conditions, such as influenza and streptococcal pharyngitis. ${ }^{8}$ This paper aims to summarize literature with respect to pharmacy-based assessment and management of cold sores to help regulators explore pharmacist prescriptive authority for oral antivirals.

\section{Considerations for Over-the-Counter or Pharmacist- Prescribed Antiviral Therapy}

Reclassification of antivirals is not a new idea. In 1994, the U.S. Food and Drug Administration (FDA) considered switching oral acyclovir to an over-the-counter product for genital herpes. ${ }^{9} \mathrm{~A}$ consensus panel met to discuss considerations for such a switch for episodic treatment of genital herpes. With respect to viral resistance, there were mixed opinions on what the outcome of over-the-counter (OTC) acyclovir would be, though the panel acknowledged "almost all acyclovir resistance has been seen in immunocompromised individuals." ${ }^{9}$ Given the safe track record of acyclovir in over 30 million people at the time, the panel concluded "the availability of OTC acyclovir would probably not be associated with significant safety problems." The biggest concern of the panel was that such a switch could set a precedent that would encourage other manufacturers of antimicrobials to seek OTC status which could drive the development of resistance, and as a result the panel 
recommended against OTC status for oral acyclovir for the treatment of genital herpes. ${ }^{9}$

Another report was published in 2012, this time evaluating the risks and benefits of moving antiviral therapies from prescription-only to a pharmacist-prescribed class of medications. ${ }^{5}$ The panel noted that the diagnoses of cold sores is based on the patient's history as well as signs and symptoms, and that laboratory confirmation is rarely needed. Given this, the panel noted that a pharmacist-prescribed antiviral move should "save the patient's time and offer greater convenience" and afford "early administration of the drug as first symptoms occur during the brief window of therapeutic opportunity." The panel reviewed the potential impact on four domains: 1) efficacy; 2 ) safety; 3 ) resistance; and 4 ) monitoring. ${ }^{5}$

With respect to efficacy, the panel noted that the increased accessibility of oral antiviral mediations in a pharmacistprescribed class might result in earlier initiation, resulting in more effective treatment and possibly prevent lesion development. The panel did not feel safety was a major concern, given that oral antivirals are "generally well tolerated and associated with minimal adverse events in patients." Similar to the previous panel, resistance was a concern, though it was noted that HSV-1 resistance remains low $(<0.5 \%)$ in immunocompetent patients, and thus the panel concluded that the development of resistance is unlikely if used episodically in immunocompetent hosts. For monitoring, the panel suggested that monitoring systems be established to ensure that patients did not self-treat genital herpes with oral antivirals, and that there were systems in place for monitoring patient safety and appropriateness of labeling. ${ }^{5}$

In addition, three surveys were identified in which Canadian pharmacists or physicians expressed opinions on pharmacistprescribed cold sore medications. In 2014, the Ontario Pharmacists Association conducted a survey of more than 200 of its members. ${ }^{10}$ Of note, $91.4 \%$ of respondents supported pharmacist prescriptive authority for minor ailments and $73.4 \%$ reported being prepared for this expanded authority. With respect to which specific conditions that pharmacists should be able to prescribe for, only four conditions were selected by more than $90 \%$ of respondents: allergic rhinitis $(93.6 \%)$, insect bites $(93.6 \%)$, cold sores $(92.9 \%)$, and canker sores $(90.7 \%) .{ }^{10}$

A second survey was conducted of 268 Saskatchewan pharmacists in 2015 , of whom $89.5 \%$ had already prescribed medication for at least one minor ailment. ${ }^{11}$ The province currently allows pharmacists to prescribe for up to 17 conditions, and pharmacists were surveyed on which conditions they felt were of least concern for patient safety. Cold sores were rated as the least concern by a large margin. ${ }^{11}$ A third study was conducted of 289 Saskatchewan physicians. ${ }^{12}$ Physicians also selected cold sores as the condition of lowest concern to patient safety with respect to pharmacist prescribing.
Select Models of Pharmacist Prescriptive Authority for Cold

\section{Sores}

\section{Canada}

Pharmacists in some Canadian provinces have had the legal authority to independently prescribe antivirals for cold sores since at least 2007..$^{13}$ In 2011, Nova Scotia and Saskatchewan created lists of "minor ailments" that pharmacists are authorized to independently prescribe for; these lists include cold sores, mild acne, allergic rhinitis, and thrush, among other ailments. ${ }^{13}$ As of 2016 , six provinces explicitly include cold sores as part of their minor ailments prescribing authority (Alberta, New Brunswick, Newfoundland, Nova Scotia, Prince Edward Island, Quebec, and Saskatchewan). ${ }^{13-15}$ This authority is classified as an autonomous, independent prescribing model. ${ }^{16}$

Studies have consistently found that cold sores are the most commonly prescribed for condition on minor ailments lists. From February 1, 2012 to December 31, 2013, cold sores accounted for 4,877 billings (49.4\%) with a total of $\$ 87,768.01$ CAD ( $\$ 17.99 /$ per episode CAD) to Saskatchewan pharmacies. ${ }^{17}$ An additional 4,453 prescribing encounters ( $45 \%$ of total encounters) were reported from January 2014 to December 2014 in Saskatchewan. ${ }^{13}$ Ninety Saskatchewan pharmacies participated in a study from September 2012 to August 2013 to evaluate patient experiences with pharmacists' prescribing for minor ailments. ${ }^{18}$ Cold sores were the most frequently treated minor ailment $(34.4 \%, n=43)$ in this study. While not specific to cold sores, patients seeking treatment at the pharmacy most commonly reported symptoms had been present just one day before visiting the pharmacy $(30.4 \%)$, and most patients (71.5\%) asked the pharmacist for help as opposed to being offered help. In addition, patients reported that had they not sought pharmacist assessment, they would have purchased an OTC medication (43.2\%) or have gone to the doctor (35.2\%), suggesting patients would either receive suboptimal therapy or misallocate resources by seeking care at a more expensive facility. ${ }^{18}$

A pilot study in Nova Scotia was conducted in 27 pharmacies from May 21, 2013 to August 16, 2013 to evaluate pharmacists prescribing for minor ailments. ${ }^{19}$ Pharmacies were reimbursed $\$ 22.50$ per patient treated for a minor ailment. A total of 1,002 had a minor ailment treated during the study period with cold sores accounting for the most frequently treated ailment $(17 \%$, $\mathrm{n}=167$ ). While not specific to cold sores, $93 \%$ of the patient assessments yielded a prescription, $96 \%$ of patients indicated the service was beneficial or very beneficial, and $89 \%$ of patients reported that their concern was satisfactorily resolved. Moreover, $96 \%$ of patients noted they were "able to access health care sooner as a result of the minor ailment service."19 No negative patient health outcomes were reported relative to cold sore management.

Consistent with the perception that cold sores were among the lowest-risk conditions for pharmacists to treat, a survey of Saskatchewan pharmacists found that very few patients 
presenting with cold sores needed a medical referral, with $30 \%$ of pharmacists saying they never facilitated a referral for this condition, and $54 \%$ estimating only a $1-10 \%$ referral rate. ${ }^{13}$

Two studies on patient experiences with pharmacy-based minor ailment services include open-ended feedback from patients. Comments relevant to prescribing for cold sores follow:

- "It is great to know that I can come in to the pharmacy to get something for my cold sore rather than waiting at the doctor's office." ${ }^{17}$

- "I am very pleased with this service. In the past, I treated cold sores with Abreva or Lipactin, which cost about \$1520 . Valtrex covered under my health benefits cost $\$ 3$ and it caused my cold sore to be much less disruptive and it was gone in 2 days." 20

\section{Australia and New Zealand}

In 2010, New Zealand re-classified famcyclovir to a "pharmacist-only" medication (POM). ${ }^{5,21}$ This move was soon followed by Australia. ${ }^{22}$ In New Zealand and Australia, medications that are classified as POM fall in an area between OTC and prescription-only drugs. While a POM can be purchased without a physician's prescription, the sale must be completed by a pharmacist and the pharmacist typically performs an assessment to ensure appropriateness of the medication prior to sale. ${ }^{23}$ Part of the rationale for the switch in New Zealand was the belief that "compliance for a single tablet dose should be higher than a cream used multiple times daily." ${ }^{22}$ No published information was found on the outcomes associated with POM famcyclovir in either county.

\section{United States}

Florida maintains a formulary of drugs that a pharmacist may dispense "within their professional judgment." ${ }^{24}$ Products are added to the formulary by a subset of members of the boards of pharmacy, medicine, and osteopathic medicine. Topical antivirals - but not oral formulations -- are a drug category included in the formulary, with penciclovir and acyclovir ointment specifically listed. Acyclovir ointment is limited to treatment of herpes simplex infections of the lip, though no such limitation is listed for penciclovir. ${ }^{24}$ While no published studies on the uptake of topical antivirals in Florida were found, these drugs have remained on the formulary since 1999 and were not removed in the 2007 update to the regulation, suggesting no significant safety issues have emerged. Florida pharmacists have, in general, not utilized this prescriptive authority to a large extent. ${ }^{25}$ Reasons for limited uptake include workflow and payment considerations. ${ }^{25}$

Idaho was the first state to allow pharmacists to independently prescribe any FDA-approved prescription for cold sores, including oral antivirals. ${ }^{26}$ The law, which took effect on July 1 , 2018, allows a pharmacist to prescribe either oral or topical antivirals for cold sores. The law initially required pharmacists to use a patient assessment protocol that specifies inclusion, exclusion, and medical referral criteria based on evidencebased research to ensure patients are appropriately triaged. Further, the law requires pharmacists to notify the patient's primary care provider of any prescription written within five business days to ensure care is not fragmented by pharmacist prescribing. ${ }^{26}$ One Idaho regional pharmacy chain noted that cold sores rank among the most sought-after service on Idaho's prescribing list; patients generally pay cash for a clinical consultation and the antiviral (when appropriate) is billed to the patient's insurer. ${ }^{27}$

\section{Discussion}

Oral antivirals for cold sores are more effective than OTC medications and have a strong safety profile. These products are routinely prescribed in Canada by pharmacists and are among the most common minor ailments for which patients seek treatment from a pharmacist. When tasked with exploring moving acyclovir to an OTC status or pharmacist-prescribed status, an expert consensus panel did not have concerns over patient safety or emergence of antiviral resistance when used in immunocompetent patients.

In general, protocols are leveraged to help pharmacists identify which patients may be safely treated in a pharmacy versus those who may need to be seen by another medical professional. Pharmacists have a history of successfully using protocols to identify appropriate candidates for treatment while referring patients when necessary because of the presence of certain high-risk factors for conditions such as influenza, strep throat, and uncomplicated urinary tract infections. ${ }^{8,28-29}$ Three such treatment protocols were found, with parameters for treatment and referral varying by jurisdiction. ${ }^{30-32}$ In addition, a standardized form is available for the pharmacist to perform the assessment for referral criteria, and to document the prescribing record. ${ }^{33-34}$

A summary of the referral criteria from these protocols is provided in Table 1. The available protocols for the treatment of cold sores are generally consistent in suggesting referral if patients are immunocompromised, if systemic symptoms are present, or if lesions appear on areas other than the mouth. A patient assessment protocol is a core element that can ensure pharmacists provide safe and effective care in accordance with evidence-based care. Further, the protocol can provide the safeguards Cunningham and colleagues described, namely guarding against the misuse of such antivirals for self-treatment of genital herpes and having a pharmacist in place to monitor safety as part of the routine follow-up care process. ${ }^{5}$

\section{Conclusion}

With the appropriate safeguards in place, pharmacists can safely assess and treat patients who have cold sores with oral antivirals as has been the case in several jurisdictions. Regulatory bodies in the United States may consider pharmacist independent prescriptive authority for oral antivirals for the treatment of cold sores as a means to improve access to patient care. 
Funding Support: None

Conflicts of Interest: None

\section{References}

1. Usatine RP, Tinitigan R. Nongenital Herpes Simplex Virus. Am Fam Physician. 2010;82(9):1075-1082.

2. Scott DA, Coulter WA, Lamey PJ. Oral shedding of herpes simplex virus type 1: a review. J Oral Pathol Med. 1997;26(10):441.

3. Johns Hopkins Medicine. Oral Herpes. Available from: https://www.hopkinsmedicine.org/healthlibrary/con ditions/adult/infectious diseases/Oral Herpes 22,Or alHerpes (Accessed January 27, 2018)

4. Embil JA, Stephens RG, Manuel FR. Prevalence of recurrent herpes labialis and aphthous ulcers among young adults on six continents. Can Med Assoc J 1975;113(7):627-30.

5. Cunningham $A$, Griffiths $P$, Leone $P$, et al. Current management and recommendations for access to antiviral therapy of herpes labialis. Journal of Clinical Virology. 2012;53:6-11. DOI: 10.1016/j.jcv2011.08.003

6. Worrall G. Herpes labialis. Clin Evid. 2009;1704.

7. Gilbert SC. Management and prevention of recurrent herpes labialis in immunocompetent patient. Herpes. 2007;14:56-61.

8. Klepser ME, Adams AJ, and Klepser DG. Antimicrobial stewardship in outpatient settings: leveraging innovative physician-pharmacist collaborations to reduce antibiotic resistance. Health Security 2015; 166-173. DOI: 10.1089/hs.2014.0083

9. Sande MA, Armstrong D, Corey L, et al. Perspectives on switching oral acyclovir from prescription to overthe-counter status: report of a consensus panel. Clin Infect Dis. 1998;26:659-63.

10. Ontario Pharmacists Association. Common Ailments Survey. September 25, 2014. Available from: https://www.opatoday.com/common ailments resul ts (Accessed September 8, 2018)

11. Taylor J, Mansell K. Minor ailment prescribing: part I pharmacist feedback. Self Care. 2016;7:10-21.

12. Taylor J. Minor ailment prescribing: part II - physician feedback. Self Care. 2016;7:22-40.

13. Taylor JG, Joubert R. Pharmacist-led minor ailment programs: a Canadian perspective. Int J Gen Med. 2016;9:291-302. DOI: 10.2147/IJGM.S99540

14. Canadian Pharmacists Association. Pharmacists' Scope of Practice in Canada. December 2016. Available from: https://www.pharmacists.ca/cphaca/assets/File/cpha-on-theissues/ScopeofPracticeinCanada DEC2016.pdf (Accessed September 8, 2018)
15. Odre des Pharmaciens du Quebec. Prescribe drugs to treat certain minor ailments. Available from: https://www.opq.org/doc/media/2225 38 frca 0 new services information sheet 4 prescribe minor ailments.pdf (Accessed September 8, 2018)

16. Adams AJ, Weaver KK. The Continuum of Pharmacist Prescriptive Authority. Ann Pharmacother.

September 2016;50(9)778-84. DOI: $10.1177 / 1060028016653608$

17. Mann J, Read E, Perrault L. Minor Ailment Prescribing in Saskatchewan. 2014. Available from:

https://www.pharmacists.ca/cphaca/assets/File/WebinarSlides-MinorAilments.pdf (Accessed September 8, 2018)

18. Mansell K, Bootsman N, Kuntz A, Talyor J. Evaluating pharmacist prescribing for minor ailments. International Journal of Pharmacy Practice. 205;23:95-101. DOI: 10.1111/ijpp.12128

19. Pharmacy Association of Nova Scotia (PANS). Evaluation of the Provision of Minor Ailment Services in the Pharmacy Setting Pilot Study. October 2013. Available from: https://pans.ns.ca/sites/default/files/2013-10-17pans-report final-exec-summary.pdf (Accessed September 8, 2018)

20. Taylor JG, Mansell K. Patient Feedback on Pharmacist Prescribing for Minor Ailments in a Canadian Province. Inov Pharm. 2017;8(1): Article 17. http://pubs.lib.umn.edu/innovations/vol8/iss1/17 DOI: https://doi.org/10.24926/21550417.1331

21. Gauld N, Kelly F, Emmerton L, et al. Innovations From 'Down-Under': A Focus on Prescription to NonPrescription Medicine Reclassification in New Zealand and Australia. Self Care. 2012;3(5):88-107.

22. Australian Government. Therapeutic Goods Administration. Famciclovir: proposed advisory statements for medicines. November 2012. Available from:

https://www.tga.gov.au/sites/default/files/consultlabelling-medicine-advisory-statements-famciclovir121109 0.pdf (Accessed September 8, 2018)

23. New Zealand Medicines and Medical Devices Safety Authority. Pharmacist-Only Medicines. Available from: http://www.medsafe.govt.nz/Consumers/PharmOnly .asp (Accessed September 8, 2018)

24. Florida Administrative Rules. 64B16-27.220. Medicinal Drugs Which May Be Ordered by Pharmacists. Available from: https://www.flrules.org/gateway/ruleno.asp?id=64B1 6-27.220 (Accessed January 27, 2018)

25. Doering PL. Prescribing Authority for Pharmacists, Florida Style: A Home Run or a Swing and a Miss? Ann Pharmacother. 2007;41:1878-83. https://doi.org/10.1345/aph.1H274 
26. Adams AJ. Pharmacist Prescriptive Authority: Lessons from Idaho. Pharmacy. 2020;8:112. DOI: $10.3390 /$ pharmacy8030112

27. Yap D. Idaho pharmacists can prescribe more than 20 categories of medications. Pharmacy Today. October 2018. Pages 44-45. Available from:

https://www.pharmacytoday.org/article/S10420991(18)31417-

8/pdf?fbclid=IwAR04noLOjysEJkIIxpUcq uC55siuJFhio s KWYqdrnh0vZp JQ7ejdbT2E (Accessed October 14, 2018)

28. Klepser ME, Adams AJ. Pharmacy-based management of influenza: lessons learned from research. International Jounral of Pharmacy Practice. 2018. https://doi.org/10.1111/ijpp.12488

29. Beahm NP, Smyth DJ, Tsuyuki RT. Outcomes of Urinary Tract Infection Management by Pharmacists (RxOUTMAP): A study of pharmacist prescribing and care in patients with uncomplicated urinary tract infections in the community. Can Pharm J. 2018;151(5):305-314. https://doi.org/10.1177/1715163518781175

30. University of Saskatchewan. medSask. Treatment Flowchart: Cold Sore (Herpes Labialis). Available from: https://medsask.usask.ca/documents/pdfs/Cold_Sore algorithm.pdf (Accessed September 8, 2018)
31. Pharmaceutical Society of Australia. Guidance for provision of a Pharmacist Only medicine Famciclovir. Available from:

https://www.psa.org.au/download/ent/uploads/fileb ase/guidelines/s3/famciclovir-protocol.pdf (Accessed September 8, 2018)

32. Chopski N. Patient Assessment Protocols. Licensees of the Idaho State Board of Pharmacy. April 12, 2018.

Available from:

https://bop.idaho.gov/code_rules/2018 04 13 Final \%20BOP\%20Protocol\%20Packet.pdf (Accessed September 8, 2018)

33. University of Saskatchewan. medSask. Pharmacist Assessment: Cold Sore https://medsask.usask.ca/documents/pdfs/Cold Sore evaluation.pdf (Accessed September 8, 2018)

34. University of Saskatchewan. medSask. Cold Sore Guidelines for Prescribing Oral Antivirals https://medsask.usask.ca/professional/guidelines/col d-sore.php (Accessed September 8, 2018) 
Table 1. Referral Criteria for Pharmacy-Managed Cold Sores

\begin{tabular}{|c|c|c|c|}
\hline Referral Criteria & Saskatchewan $^{30}$ & Australia $^{31}$ & Idaho $^{32}$ \\
\hline Patient Age & Not addressed & Refer if $<18$ years & Refer if $<6$ years \\
\hline Patient Immune Status & Refer if immunocompromised & Refer if immunocompromised & Refer if immunocompromised \\
\hline Systemic Symptoms & $\begin{array}{l}\text { Refer if "fever, swollen glands } \\
\text { or other systemic symptoms" }\end{array}$ & Refer if "Systemic symptoms present" & $\begin{array}{l}\text { Refer if "Symptoms of systemic } \\
\text { illness are present (fever, swollen } \\
\text { glands, malaise)" }\end{array}$ \\
\hline Lesion Duration & $\begin{array}{l}\text { Refer if present }>14 \text { days or if } \\
\text { "lesion does not completely } \\
\text { heal between episodes" }\end{array}$ & Refer if present $>7$ days & Can only treat if lesion $<48$ hours \\
\hline Lesion Location & $\begin{array}{l}\text { Refer if "lesion on or around } \\
\text { nose" }\end{array}$ & $\begin{array}{l}\text { Refer if "lesions on other parts of the } \\
\text { body" }\end{array}$ & $\begin{array}{l}\text { Refer if "lesion appears on area } \\
\text { other than around the mouth and } \\
\text { lips" }\end{array}$ \\
\hline Lesion Features & $\begin{array}{l}\text { Refer if "lesion excessively } \\
\text { red, swollen, or contains pus" }\end{array}$ & $\begin{array}{l}\text { Refer if "signs of bacterial skin } \\
\text { infection" or if there is "Painful mouth } \\
\text { ulceration with poor oral intake" }\end{array}$ & $\begin{array}{l}\text { Refer if "lesion appears excessively } \\
\text { red, swollen, or contains pus" }\end{array}$ \\
\hline Frequency & Refer if $>6$ episodes per year & Refer if $>3$ episodes per year & Refer if $>6$ episodes in 12 months \\
\hline Follow-up & $\begin{array}{l}\text { Follow-up at day } 7 \text { and refer if } \\
\text { no signs of response }\end{array}$ & $\begin{array}{l}\text { Follow-up at day } 7 \text { and refer if no } \\
\text { signs of response }\end{array}$ & $\begin{array}{l}\text { Follow-up at day } 7 \text { and refer if } \\
\text { lesions spread or persist without } \\
\text { improvement }\end{array}$ \\
\hline
\end{tabular}

